\title{
Arthritis in Lewis rats induced by the non-immunogenic adjuvant CP20961: an immunohistochemical analysis of the developing disease
}

\author{
S C R Meacock, D R Brandon, M E J Billingham
}

\begin{abstract}
Objectives-The role of lymphocytes and macrophages in developing adjuvant arthritis induced by an injection of CP20961 in inbred Lewis rats was studied over a 32 day period using a novel biotinavidin immunoperoxidase histochemical technique.

Methods-Fresh frozen sections of hind paws and spleens, as well as lymph nodes draining the site of the injected adjuvant were immunostained using a panel of monoclonal antibodies specific for subsets of lymphocytes and macrophages and for MHC Class II antigen.

Results-An increase in the numbers of activated T-lymphocytes was detected early in the draining lymph nodes before hind paw swelling had begun. The presence of these cells in significant numbers was only observed in the vicinity of the joint after joint swelling and damage had begun. Macrophages were among the first cells to invade the swollen paws and later were found with T-lymphocytes and cells bearing the MHC class II antigen at the face of eroding and re-organising bone.

Conclusions-The activity of T-lymphocytes in initiating arthritis appeared to occur early in lymph nodes. Joint destruction was more closely associated with the arrival of macrophages but later arrival of T-lymphocytes may have contributed to the maintenance of chronic inflammation.
\end{abstract}

(Ann Rheum Dis 1994; 53: 653-658)

Lilly Research Centre Limited,

Windlesham, Surrey, United Kingdom $S$ C R Meacock D R Brandon

ME J Billingham

Correspondence to: Dr M Billingham, University of Bristol, Department of Medicine, Rheumatology Unit, Rheumatology Unit,
Bristol Royal Infirmary,
Bristol BS2 8HW, Bristol BS2 8HW,

Accepted for publication 27 April 1994

Polyarthritis has been induced in rats by injection in oil of several immunogenic preparations such as mycobacteria, streptococcal cell walls and collagen Type II. ${ }^{1}$ The non-immunogenic synthetic adjuvant, CP20961, has also been used to induce and time course of developing disease. ${ }^{2}$ As with mycobacterial adjuvant disease, arthritis could be transferred to inbred rats of the same strain with lymphocytes from diseased animals ${ }^{3}$ indicating that, regardless of the immunogenic status of the arthritogen, lymphocytes were important in the initiation of arthritic disease.
In the case of CP20961, this has led to the proposal that it causes non-specific activation of immunity to an autoantigen, latent virus or viral protein, perhaps by activation of antigenprocessing cells or by intensifying the traffic in local lymph nodes draining the site of initiating injectant. $^{2}$

The histopathology of CP20961 arthritis has been studied previously and found to resemble very closely that of other rat models of arthritis such as collagen Type II and mycobacterial adjuvant disease, ${ }^{12}{ }^{4}$ though this does not infer a similar induction mechanism and pathogenesis for these differing arthritogens. With the advent of a range of monoclonal antibodies (MAbs) to various rat leucocyte markers the opportunity arose to examine more specifically the kinetics of the arrival of different cells in the lesions of paws from diseased animals and to monitor changes which occur in their lymphoid tissue. With this study in view a biotin-avidin immunohistoperoxidase technique to stain sections of unfixed whole joints was developed, ${ }^{5}$ which could withstand the application of successive multiple reagents and retain good morphology.

With this method a panel of MAbs diagnostic for lymphocytes, macrophages and MHC Class II antigen was used to immunostain sections of hind paws, spleens and lymph nodes draining the site of injection during the first 32 days of developing disease. The results indicate that lymphocytes are late arrivals in inflamed joints and are therefore unlikely to initiate joint and soft tissue destruction directly and are more likely to play their initiating role in lymphoid tissue. The strongest candidate for the initiator of destruction in the joint is the macrophage. The opportunities for this cell to interact with endogenous and other invading cells is discussed. arthritis with a very similar clinical appearance

\section{Materials and methods}

CP20961 ARTHRITIS

Female inbred Lewis rats $(140-160 \mathrm{~g}$, Olac, Bicester, UK) were injected intradermally at the tail base with $5 \mathrm{mg}$ of CP20961 (Avridine; Pfizer, Groton, CT [N,N-dioctadecyl-N',N'-bis (2-hydroxy-ethyl) propanediamine]) in $0.1 \mathrm{ml}$ paraffin oil. ${ }^{6}$ Groups of five animals were sacrificed on days $4,7,11,14,18,21,25$ and 32 after initiation of disease together with age 
matched controls, and their hind paws were dissected above the ankle joint. After removal of the skin the paws from three animals were snap-frozen in isopentane at $-70^{\circ}$ and stored at $-80^{\circ}$ until used for immunohistochemistry; the remainder were used for conventional histopathology. Spleens and draining lymph nodes from the same animals were collected, and used similarly.

\section{IMMUNOHISTOCHEMISTRY}

Immunohistochemical staining of cryostat sections $(10 \mu \mathrm{m})$ of rat paws was performed according to the immunohistoperoxidase technique of Meacock et $a l^{5}$ using the monoclonal antibodies (MAb) listed in table 1 to stain adjacent tissue sections. Briefly, the various immunochemical reagents were added successively to the sections attached to the adhesive side of transparent sellotape fixed horizontally, by one end, on microscope slides in a perspex humidity cabinet at $4^{\circ}$. Mild formalin fixation and blocking of endogenous peroxidase was performed after the incubation steps with $\mathrm{MAb}$ and biotinylated anti-mouse IgG conjugate and before the addition of horseradish peroxidase-avidin $\mathrm{D}$. The colour reaction was developed with tetraaminobiphenyl hydrochloride (BDH, Poole, Dorset, UK) and the blocking of endogenous peroxidase was preserved by immersing in citric acidphosphate buffer, $\mathrm{pH} 2 \cdot 3$. Finally, the sections were counterstained lightly with Mayer's haematoxylin and mounted in Apathy's medium. Controls included the use of an irrelevant antiserum and replacement of the primary $\mathrm{MAb}$ and biotinylated $\mathrm{IgG}$ with diluent buffer. Further control sections were stained with haematoxylin and eosin $(\mathrm{H} \& \mathrm{E})$.

Cryostat sections $(4 \mu \mathrm{m})$ of spleen and lymph nodes were mounted on poly-l-lysinecoated multispot slides, allowed to dry for one hour and fixed in acetone for five minutes at $4^{\circ}$. The sections were then stained according to the immunohistoperoxidase technique previously described. ${ }^{16}$ Control sections were stained with $\mathrm{H} \& \mathrm{E}$.

\section{OTHER REAGENTS}

Mouse MAbs directed against rat macrophages (ED1, ED2, ED3), MHC Class II antigen (OX-6), B-lymphocytes (OX-33) and T-lymphocytes (OX-19, R73) were purchased from Serotec, Oxford, UK. MAbs directed against subsets of rat T-lymphocytes (OX-35 and $\mathrm{OX}-8$ ) were purified by protein A chroma-

Table 1 Monoclonal antibody panel

\begin{tabular}{|c|c|c|c|}
\hline Antibody & Isotype & Specificity & Reference \\
\hline OX -35 & IgG2a & T-helper lymphocytes, macrophages & 7 \\
\hline $0 X-8$ & IgG1 & T-suppressor/cytotoxic lymphocytes & 8 \\
\hline OX-19 & IgG1 & Pan T-lymphocytes & 9 \\
\hline OX-33 & IgG1 & LCA, only on B lymphocytes & 10 \\
\hline OX-6 & IgG1 & RT1B (Class II Monomorphic) & 11 \\
\hline ED1 & IgGl & Monocytes, macrophages & 12 \\
\hline ED2 & IgG1 & Macrophages & 12 \\
\hline ED3 & IgG2a & Macrophages & 12 \\
\hline R73 & $\mathrm{IgGl}$ & $\alpha / \beta$ T lymphocyte receptor & 13 \\
\hline NDS64 & IgG2b & IL-2R, p55 & 14 \\
\hline OX -39 & IgG1 & IL-2R, p55 & 15 \\
\hline
\end{tabular}

tography from ascites fluids using hybridomas kindly provided by Dr Alan Williams (MRC Cellular Immunology Group, Oxford University, UK). MAbs directed against IL-2R, p55 (OX-39, NDS64) were a gift from Dr M J Dallman, John Radcliffe Hospital, Oxford, UK. Biotinylated horse anti-mouse IgG, affinity purified, and horseradish peroxidase-avidin D were purchased from Vector laboratories, Peterborough, Cambs, UK. Scotch Brand 800, a polyvinyl acetate sellotape was purchased from $3 \mathrm{M}$ industries, Bracknell, Berks, UK.

\section{Results}

STANDARD HISTOPATHOLOGY

Sections stained with $H \& E$ indicated that the hind paws remained normal until day 11 when small pockets of oedema fluid containing polymorphonuclear and mononuclear cells were seen in soft tissue adjacent to the tibia and calcaneus. By day 14 the paws were grossly swollen with greater numbers of infiltrating cells often forming clusters in the soft tissue and synovial membranes. From day 18 onwards intense synovitis and pannus formation developed (fig 1A) together with periostitis which led to subchondral bone invasion, erosion and reorganisation. Infiltration by mononuclear cells of areas of ligament attachment became evident and pannus overgrowth of cartilage eventually was associated with cartilage destruction on days 25 and 32 .

Disease progression caused lymphoid hypertrophy earlier than the appearance of inflammation in the joints. An increase in cell numbers in the paracortical areas of the draining lymph nodes was seen as early as day 4 , as well as the appearance of neutrophils and eosinophils in the red pulp of the spleen by day 11 . Eventually the ratio of the sizes of the red pulp to the white pulp in the spleen became obviously greater.

\section{IMMUNOHISTOLOGY OF NORMAL CONTROL}

PAWS

Table 2 summarises the staining patterns of sections of normal paws. It shows that the Tand B- cell markers rarely stained cells in soft tissue adjoining bone or in the synovium. The positive staining by $\mathrm{OX}-35$, a marker for monocytes and macrophages as well as T-helper lymphocytes, paralleled almost completely the staining pattern for the macrophage markers ED1 and ED2. ED3 labelled only an occasional cell in the synovial lining and bone marrow with its characteristic pale staining. OX-6, the marker for MHC Class II antigen, stained cells in loose connective tissue adjacent to tendons and ligaments and periosteum as well as cells in the stromal layer of the bone marrow interface with cancellous bone.

IMMUNOPATHOLOGY OF PAWS FROM ANIMALS WITH DEVELOPING DISEASE

Lymphocyte markers: Increased numbers of cells staining with the T-lymphocyte markers OX-8, 


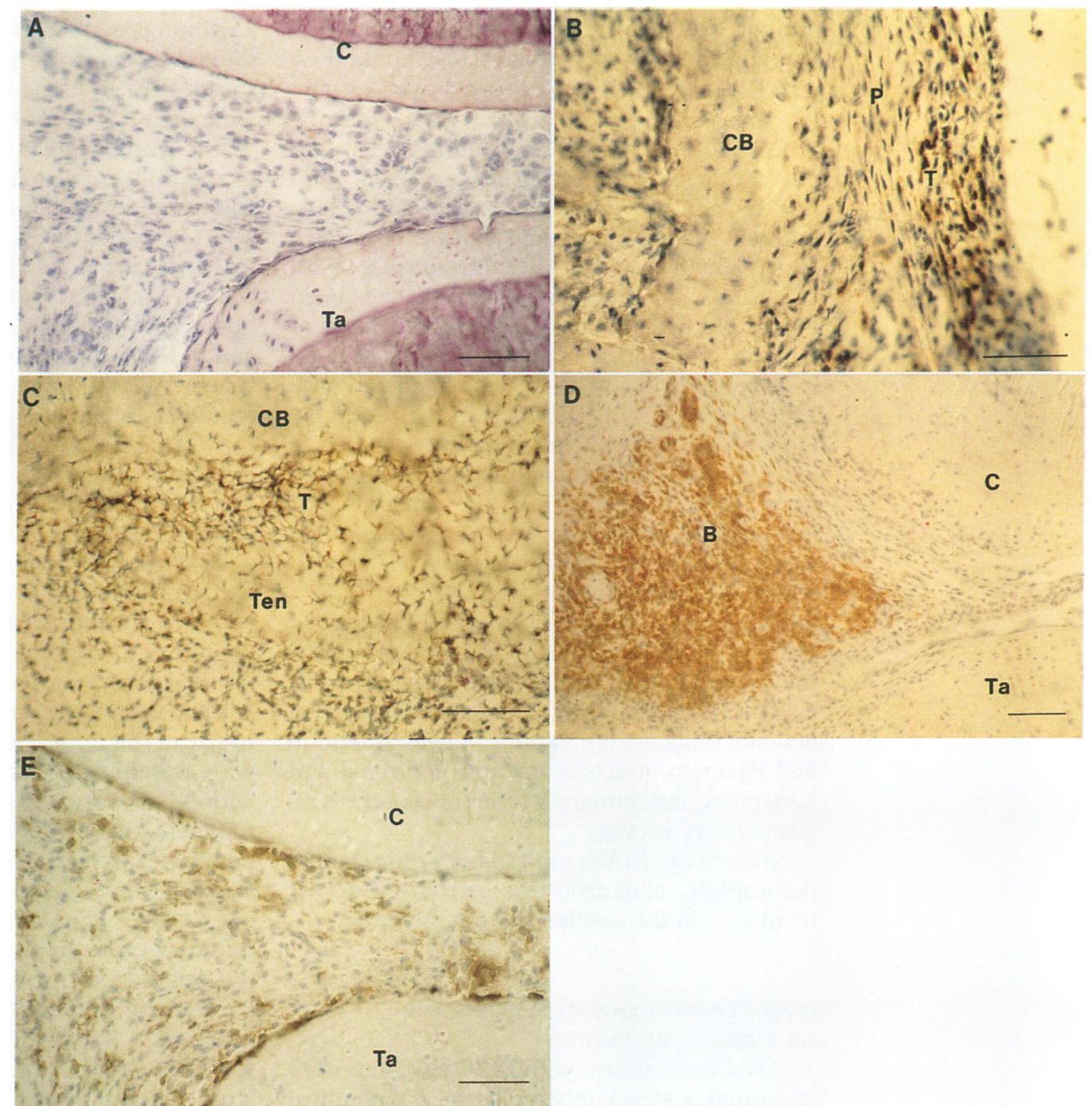

Figure 1 Adjuvant arthritis. (A) Day 18. Synovium infiltrated with mononuclear cells and some polymorphonuclear cells, covering cartilage of talus and calceneous, both with loss of chondrocyte staining. (Haematoxylin and eosin staining.)

(B) Day 32. T-cells labelled with OX-19 in inflamed synovium adjacent to periosteum. (C) Day 18. T-cells labelled with $R 73$ at the attachment point of a tendon with bone. (D) Day 25 . Large numbers of B-cells labelled with OX-33 in the subsynovium between the talus and calcaneus. (E) Day 18. Adjacent section to fig $1 A$. Strong labelling of synovial lining and sublining cells with ED1. A similar staining pattern was seen at this time with ED2 and ED3. C, calcaneous; Ta, talus; $C B$, cortical bone; Ten, tendon; $P$, periosteum; $T, T$-cells; B, B-cells. Bar $=25 \mu \mathrm{m}$.

Table 2 Staining pattern ${ }^{\star}$ of sections of normal rat hind paws

\begin{tabular}{|c|c|c|c|c|c|c|c|c|c|c|}
\hline$M A b$ & $O X-35$ & $O X-8$ & $O X-19$ & $R 73$ & $\begin{array}{l}N D S 64 \\
\text { \& OX-39 }\end{array}$ & $O X-33$ & $E D 1$ & $E D 2$ & $E D 3$ & $O X-6$ \\
\hline Synovial lining cells & + & - & - & - & - & - & + & + & \pm & \pm \\
\hline $\begin{array}{l}\text { Sub Synovial lining } \\
\text { cells }\end{array}$ & + & - & \pm & \pm & \pm & - & + & \pm & - & \pm \\
\hline $\begin{array}{l}\text { BM cells at bone } \\
\text { interface }\end{array}$ & + & - & - & - & - & - & + & + & \pm & ++ \\
\hline Other BM cells & + & \pm & + & + & - & \pm & + & + & - & + \\
\hline Periosteal cells & \pm & - & - & - & - & - & \pm & \pm & - & \pm \\
\hline $\begin{array}{l}\text { Interstitial muscle } \\
\text { cells }\end{array}$ & + & - & - & - & - & - & & - & - & \\
\hline $\begin{array}{l}\text { Tendon/ligament } \\
\text { cells }\end{array}$ & - & - & - & - & - & - & \pm & \pm & - & - \\
\hline $\begin{array}{l}\text { Loose connective } \\
\text { tissue cells }\end{array}$ & + & - & \pm & \pm & \pm & \pm & + & + & - & + \\
\hline $\begin{array}{l}\text { Interstitial cells in } \\
\text { nerve cells }\end{array}$ & + & - & - & - & - & - & + & - & - & - \\
\hline
\end{tabular}

$\star \pm$, rarely present; +, present; ++, present and strongly stained.

OX-19, R73 and NDS $64+$ OX-39 were not seen in synovium or in inflammatory infiltrates until day 18 , although occasionally a few cells were seen scattered randomly at earlier times in soft tissue. Cells staining with OX-35 on days 11 and 14 were morphologically more like macrophages than lymphocytes (see below). From day 18 onwards increasing numbers of stained T-lymphocytes were found with infiltrating macrophages in the synovium below the outer lining (fig $1 \mathrm{~B}$ ), in clusters in soft tissue and at tendon and ligament attachment points (fig $1 \mathrm{C}$ ). In addition, larger numbers of labelled cells were found in the bone marrow of reorganising bone.

Large infiltrates of cells, stained with OX-33 and therefore presumably B lymphocytes, were consistently found in the subsynovium adjacent to the talus and calcaneus from day 18 onwards (fig 1D). Cells labelled with OX-33 were also found scattered in soft tissue, often in clusters.

Macrophage markers: Mononuclear cells first seen in oedema fluid on day 11 stained with OX-35 and ED1 and very rarely with ED2. By day 14 increased staining with all three MAbs of synovial lining and sublining cells (fig 1E), bone marrow cells and infiltrating macrophages in soft tissue was evident. From day 18 onwards infiltrating cells at ligament and tendon attachment points were heavily stained as well as cells at invagination areas into eroding bone or streaming into bone marrow 
through the periosteum adjacent to cartilage. Staining with ED3 was always less intense than with ED1 and ED2 and appeared to be specific for macrophages or their bone marrow precursor cells. Numbers of stained cells were not significantly increased above normal until day 18 onwards when they were principally found in synovial lining, in swirls of cells in soft tissue, and in bone marrow at the interface with cancellous bone in areas which had been invaded by inflammatory cells. Examination of adjacent sections suggested that not all macrophages in soft tissue which stained with EDI stained with ED3 (fig 2A and B). Conversely, it was not possible to decide whether some cells stained with ED3 and not with ED1. With regard to macrophage labelling by $\mathrm{OX}-35$, it was noted that some swirls of cells in soft tissue, heavily labelled with ED2, did not label with OX-35.

MHC Class II Antigen: From day 11 increased staining by OX-6 of cells in areas stained by macrophage and lymphocyte markers was evident. For example, mononuclear cells in oedema fluid, accumulations of cells at tendon and ligament attachments, and periosteal and juxtaposed inflammatory mononuclear cells all stained very strongly.

None of the MAbs used in this study stained neutrophils, chondrocytes, osteocytes or osteoid cells in the swollen paws at any time.

IMMUNOPATHOLOGY OF SPLEENS AND DRAINING LYMPH NODES

The staining patterns with ED1, ED2 and ED3 of normal control lymphoid tissue was identical to that reported by Dijkstra et al. ${ }^{12}$ The
T-lymphocyte markers OX-8, OX-19 and R73 stained areas of the periarteriolar lymphocyte sheath (PALS) of the spleen white pulp and the paracortical areas of lymph nodes as expected. The B-lymphocyte marker OX-33 stained cells in the follicle and marginal zone areas of the spleen and in the cortex of lymph nodes. OX-35 stained cells in the PALS as well as macrophages in the red pulp of the spleen and cells in the paracortex and medulla of lymph nodes. OX- 6 stained diffusely in the red pulp and in B-lymphocyte areas of the white pulp of spleen, as well as cells in the cortex and medullary areas of lymph nodes. NDS64 and OX-39, used sometimes in combination to enhance staining, stained only a few cells in the PALS of normal spleen and the paracortex of lymph nodes.

With the development of disease there was no significant change in pattern of staining with the macrophage markers, most of the lymphocyte markers or OX-6. ED2, since it stains macrophages mainly in the red pulp of spleen, served to confirm the relative increase in size of this area. The most significant change with developing disease, however, was seen with NDS64 and OX-39. By day 4 (fig 3A) the numbers of lymphocytes staining in the paracortex of the draining lymph nodes had started to increase and were maintained until day 14 after which they declined. In the spleen numbers of stained lymphocytes in the PALS did not start to increase until day 11(fig 3B), particularly at the interface with the marginal zone and started to decline on day 21 . Eosinophils seen in the red pulp stained strongly with these anti-IL-2R reagents, as previously reported. ${ }^{16}$
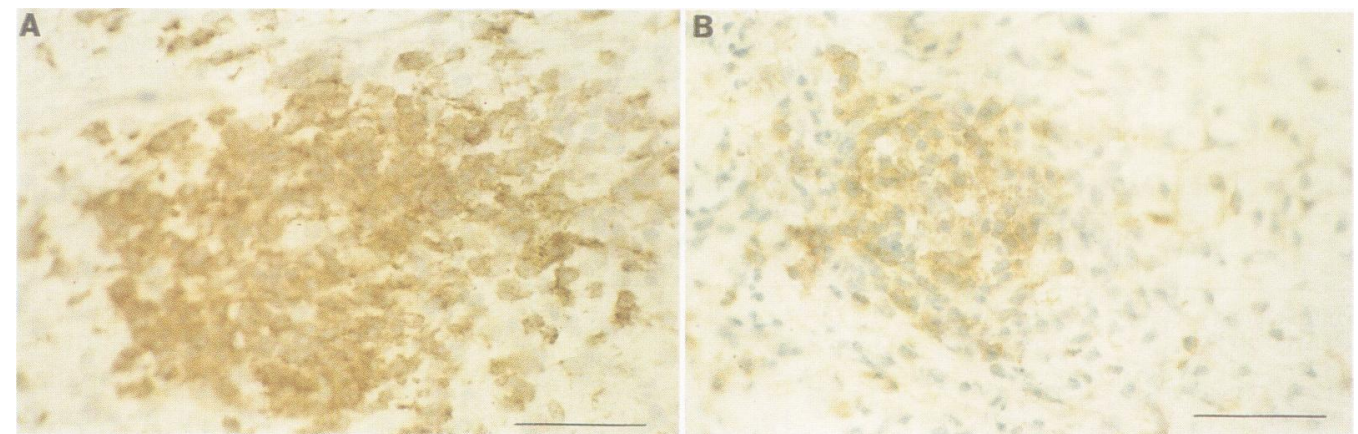

Figure 2 Adjuvant arthritis Day 18. A cluster of cells surrounding a venule in soft tissue labelled with $(A)$ ED1 and (B) $E D 3$. Bar $=25 \mu m$.

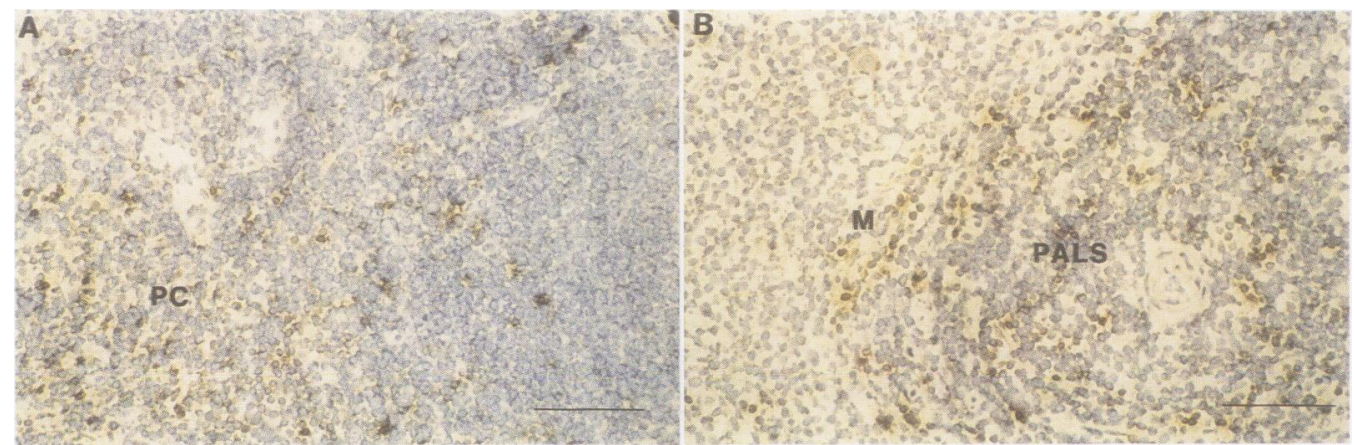

Figure 3 Adjuvant arthritis. Lymphocytes labelled with $O X-39$ in $(A)$ the paracortex $(P C)$ of a draining lymph node on day 4 and $(B)$ the PALS and marginal zone $(M)$ of a spleen on day 11. Bar $=25 \mu \mathrm{m}$. 


\section{Discussion}

Immunohistochemical staining of sections of paws from normal Lewis rats with the present panel of MAbs indicated that very few cells were stained with the markers specific for lymphocytes. Occasionally, mainly with OX-19, cells were seen in the synovium below the outer lining or in the bone marrow. Stronger labelling of cells was seen, however, with the macrophage markers ED1 and ED2, which labelled some synovial lining cells and cells in loose connective tissue and bone marrow, but labelling of normal tissue with ED3 was virtually absent. OX-6, the marker for MHC Class II antigen stained strongly the stromal layer of cells interfacing the bone marrow and cancellous bone as well as some cells in loose connective tissue close to the periosteum.

CP20961 was chosen as the adjuvant to induce arthritis as it is non-antigenic and does not persist, unlike mycobacteria which are antigenic and remain in the rat for several weeks. The presence of persistent mycobacteria would complicate the interpretation of cell traffic into the arthritic lesion sites. With the development of disease, immunostaining intensity increased gradually. No attempt was made to double label sections, but examination of adjacent sections with different MAbs suggested that macrophages in oedema fluid labelled predominantly with ED1 or OX-35, and that increases in ED2 and ED3 labelling occurred later than with ED1 as has been seen previously in antigen-induced arthritis. ${ }^{17}$ Furthermore, not all cells which stained with ED1 stained with ED3. This supports the view of Beelen et $a l^{18}$ that ED1, ED2 and ED3 reflect different stages of differentiation of macrophages which may be affected by factors released during the inflammatory process.

It was intriguing to note that one area of synovium in particular, adjacent to the talus and calcaneus, labelled strongly with the B-lymphocyte marker OX-33 and only sparsely with T-lymphocyte markers. It is not clear why this should have occurred, but possibly these cells were involved in autoantibody production.

The time course of developing CP20961 arthritis was very similar to that originally observed by Chang et al, ${ }^{2}$ also in Lewis rats. The first signs of swollen paws was on day 11 . The immunohistopathology was very consistent from animal to animal, as has been shown with this model previously in terms of arthritic score. ${ }^{19}$ Macrophages, stained by ED1 or OX-35, and polymorphonuclear cells were found on days 11 and 14 in growing pockets of oedema fluid adjacent to the ankle joint and in the surrounding soft tissue. Only the occasional cell in soft tissue or synovium was stained with the lymphocyte markers OX-19, OX-8 and R73 and it is unlikely therefore that T-lymphocytes had the opportunity to contribute directly to the initiation of joint inflammation occurring at that time. From day 18 onwards the predominant invading cells were mononuclear, staining with either macrophage or T-lymphocyte markers, and were found often in the same locality in joint synovium, at the interface with eroding bone and at tendon and ligament attachment points. Since staining with the MHC Class II antigen marker OX-6 was also enhanced in these areas the opportunity to process and present putative autoantigen to stimulate the local release of destructive enzymes was evident. Recent results by Meijers et $a l^{19}$ lend support to this view. They have shown in the same disease model that the local production of the cysteine proteinases Cathepsins B and L occurred in areas of eroding bone and cartilage invaded by macrophages which had been stained by ED1 or ED2. Enzyme levels reached a maximum 20 days after induction of arthritis and decreased as the disease entered natural remission.

Examination of lymphoid tissue from diseased animals revealed the early production in lymph nodes draining the site of injection of CP20961 of T-lymphocytes bearing IL-2 receptors. Increased numbers of cells were seen as early as day 4 and were maintained until day 14 after which cell numbers decreased. A similar wave occurred later in the spleen on day 11 and was followed by an apparent decline on day 21. Possibly these activated lymphocytes were part of the lymphocyte population involved in the initiation stages of the disease, which have been shown capable also of generating arthritis in normal animals by adoptive transfer. ${ }^{3}$ However, even in the case of adoptive transfer of arthritis with arthritogenic T-lymphocytes, none of these were found to traffic to lesion sites when labelled with fluorochromes; lymph nodes, spleen and liver were the primary sites of traffic. ${ }^{20}$

Billingham et $a l^{21}$ have previously shown that prophylactic treatment with OX-35 inhibited the development of adjuvant arthritis and postulated that modulation of disease was due to depletion of $\mathrm{T}$-helper cells or by regulation of their function. In the present study cell labelling by $\mathrm{OX}-35$ supported this hypothesis. However, since labelled macrophages were also found in lymphoid tissue and diseased joints it was possible that therapy with OX-35 could have affected macrophage numbers or function in the study by Billingham et al. However, Yoshino et $\mathrm{al}^{22}$ showed that R73, a Mab specific for the $\alpha / \beta T$-cell receptor, also inhibited adjuvant arthritis and therefore it is likely that the major effect of OX-35 was on T-helper cells.

In conclusion, using a panel of MAbs specific for various subsets of lymphocytes and macrophages it has been possible to identify different stages of disease development and to comment on the possible role of these cells. Macrophages bearing the ED1 marker were among the first cells to arrive in the swollen paws and were the principal invading cells. These were followed by more mature macrophages bearing the ED2 and ED3 markers. Opportunity for them to interact with T-lymphocytes and MHC Class II antigenbearing cells in synovium, at ligament attachment points or at the face of eroding bone was evident.

The activity of T-lymphocytes in initiating CP20961 arthritis appeared to occur early in 
the draining lymph nodes and not directly in the paw since these cells only arrived in significant numbers after joint swelling and damage had begun. At later times, however, they may have contributed to the maintenance and, possibly, resolution of the inflammatory process.

We thank Mr C P Brown and Mrs Caroline Hicks for their expert technical assistance and Mrs Sue Donovan for her careful typing of the manuscript.

1 Billingham M E J. Models of arthritis and the search for anti-arthritic drugs. In: Orme M C L'E, ed. Anti-Rheumatic Drugs. New York: Pergamon Press, 1990: $1-47$.

2 Chang Y H, Pearson C M, Abe C. Adjuvant arthritis. IV. Induction by a synthetic adjuvant: immunologic, Induction by a synthetic adjuvant. immunologic, 23: $62-71$.

3 Taurog J D, Sandberg G P, Mahowald M L. The cellula basis of adjuvant arthritis. II. Characterisation of the cells mediating passive transfer. Cell Immunol 1983; 80 198-204

4 Pearson C M, Wood F D. Studies of arthritis and other lesions induced in rats by the injection of mycobacterial adjuvant. VII. Pathologic details of the arthritis and spondylitis. Am f Path 1963; 42: 73-95.

5 Meacock S C R, Brandon D R, Brown C P, Swann B P. A novel technique for immunohistoperoxidase staining of A novel technique for immunohistoperoxidase staining of unfixed whole jo

6 Billingham M E J, Carney S, Butler R, Colston M J. A mycobacterial $65-\mathrm{kD}$ heat shock protein induces antigenspecific suppression of adjuvant arthritis, but is not itsel arthritogenic. $\mathcal{F} \operatorname{Exp} M e d$ 1990; 171: 339-44.

7 Jefferies W A, Green J R, Williams A F. Authentic T helper CD4 (W3/25) antigen on rat peritoneal macrophages. f Exp Med 1985; 162: 117-27.

8 Brideau R J, Carter P B, McMaster W R, Mason D W, Williams A F. Two subsets of rat T lymphocytes defined with monoclonal antibodies. Eur f Immunol 1980; 10: with mon-15.

9 Dallman M J, Mason D W, Webb M. The roles of host and donor cells in the rejection of skin allografts by T-celldeprived rats injected with syngeneic $\mathrm{T}$ cells. Eur $\mathcal{f}$ Immunol 1982; 12: 511-8.
10 Woolett C R, Barclay A N, Puklavec M, Williams A F. Molecular and antigenic heterogeneity of the rat leukocyte-common antigen from thymocytes and $\mathrm{T}$ and B lymphocytes. Eur f Immunol 1985; 15: 168-73.

11 McMaster W R, Williams A F. Identification of Ia glycoproteins in rat thymus and purification from rat spleen. Eur f Immunol 1979; 9: 426-33.

12 Dijkstra C D, Dopp E A, Joling P, Kraal G. The heterogeneity of mononuclear phagocytes in lymphoid organs: distinct macrophage subpopulations in the rat recognized by monoclonal antibodies ED1, ED2 and ED3. Immunology 1985; 54: 589-99.

13 Hunig $T$, Wallny H-J, Hartley J K, Lawetzky A Tiefenthaler G. A monoclonal antibody to a constan determinant of the rat $T$ cell antigen receptor that induces $\mathrm{T}$ cell activation. Differential reactivity with subsets of immature and mature lymphocytes. 7 Exp Med 1989; 169; 73-86.

14 Tellides G, Dallman M J, Morris P J. Mechanism of action of Interleukin-2 receptor (IL-2R) monoclonal antibody (MAb) therapy: target cell depletion or inhibition of function? Transpl Proc 1989; 21: 997-8.

15 Patterson D J, Jefferies W A, Green J R, et al. Antigens of activated rat $\mathrm{T}$ lymphocytes including a molecule of $50000 \mathrm{Mr}$ detected only on CD4 positive T blasts. Mol Immunol, 1987; 24: 1281-90.

16 Meacock S C R, Brandon D R, Smith W. Interleukin-2 receptors on rat eosinophils in adjuvant arthritis. Immunology 1991; 74: 169-71.

17 Verschure P J, Van Noorden C J F, Dijkstra C D. Macrophages and dendritic cells during the early stages of antigen-induced arthritis in rats: immunohistochemical analysis of cryostat sections of the whole knee joint. Scand Immunol 1989; 29: 371-81.

18 Beelen R H J, Eestermans I L, Dopp E A, Dijkstra C D. Monoclonal antibodies ED1, ED2, and ED3 against rat macrophages: Expression of recognized antigens in different stages of differentiation. Transplant Proc 1987 19: $3166-70$

19 Meijers M H M, Kool J, Meacock S C R, Van Noorden C J F, Billingham M E J. Cysteine proteinase activity in the development of arthritis in an adjuvant model in the rat. Agents $\mathcal{E}$ Actions 1993; 39: C219-21.

20 van de Langerigt A G M, Volsen S G, Hicks C A Craig P J, Billingham $M$ E J, van den Berg W B. Cell migration studies in the adoptive transfer of adjuvant arthritis in the rat. Immunology 1994 (in press).

21 Billingham M E J Hicks C, Carney S. Monoclonal antibodies and arthritis. Agents $\&$ Actions 1990; 29: 77-87.

22 Yoshino S, Schlipkoter E, Kinne R, Hunig T. Suppression and prevention of adjuvant arthritis in rats by a monoclonal antibody to the $\alpha / \beta \mathrm{T}$ cell receptor. Eur $\mathcal{F}$ Immuno 1990; 20: $2805-8$. 